\title{
An observational pursuit for Pop III stars in a Ly $\alpha$ emitter at $z>6$ through He II emission
}

\author{
Tohru Nagao \\ National Astronomical Observatory of Japan, Mitaka, Tokyo 181-8588, Japan \\ email: tohru@optik.mtk.nao.ac.jp
}

\begin{abstract}
We report our on-going observational project to search for population III (Pop III) stars in high- $z$ galaxies. We searched Ly $\alpha$ emitters (LAEs) with a large equivalent width (EW), by our new selection technique 'NB921-depressed $i$ '-dropout selection'. We found eight photometric candidates and spectroscopically identified five LAEs with $E W_{0}(\operatorname{Ly} \alpha)>100 \AA \dot{W}$ e then carried out a very deep near-infrared spectroscopy for a LAE among the above five, to search for the redshifted He II $\lambda 1640$ emission from Pop III stars in the galaxy, but obtained only an upper limit.
\end{abstract}

Keywords. early Universe, galaxies: evolution, galaxies: starburst, stars: early

The detection and investigation of the first-generation stars, Population III (PopIII) stars, will be one of the main goals of astronomy in the next decade. Since galaxies with massive Pop III stars are expected to show a very strong Ly $\alpha$ emission and a detectable He II emission in their spectra (e.g., Schaerer 2002; Schaerer 2003), we are promoting a project to search for such spectroscopic signatures of Pop III stars.

We developed a new method to select Ly $\alpha$ emitters (LAEs) with a large equivalent width (EW) at a wide redshift range, $6.0<z<6.5$, by focusing 'NB921-depressed $i^{\prime}$ dropout' objects (Nagao et al. 2004). Through the follow-up spectroscopic observations with Subaru and Keck telescopes, we identified five strong LAEs with $E W_{0}(\operatorname{Ly} \alpha)>100 \AA$ among eight photometric candidates (Nagao et al. 2005; Nagao et al. 2007).

Among the identified NB921-depressed $i^{\prime}$-dropout galaxies, we focused on a LAE at $z=6.33$ and with $E W_{0}(\operatorname{Ly} \alpha)=130 \AA$ and carried out a very deep $J$-band spectroscopic observation to search for the redshifted He II $\lambda 1640$ emission from Pop III stars in this LAE. Even after $42 \mathrm{ksec}$ of integration with the Subaru-OHS spectrograph, no emission-line features are detected in the $J$-band. We obtained a $2 \sigma$ upper limit of $9.06 \times 10^{-18} \mathrm{erg} \mathrm{s}^{-1} \mathrm{~cm}^{-2}$ on the He II $\lambda 1640 \mathrm{flux}$, which corresponds to a luminosity of $4.11 \times 10^{42} \mathrm{erg} \mathrm{s}^{-1}$. This upper limit implies that the upper limit on the Pop III star formation rate is in the range $4.9-41.2 \mathrm{M}_{\odot} \mathrm{yr}^{-1}$ if Pop III stars suffer no mass loss, and in the range $1.8-13.2 \mathrm{M}_{\odot} \mathrm{yr}^{-1}$ if strong mass loss is present. The non-detection of He II in the target LAE may thus disfavor weak feedback models for Pop III stars.

\section{Acknowledgements}

This study was done under a large collaboration and the names of collaborators cannot be given in the author list due to the limited space. TN is financially supported by JSPS.

\section{References}

Nagao, T., Taniguchi, Y., Kashikawa, N., et al. 2004, ApJ (Letters), 613, L9

Nagao, T., Kashikawa, N., Malkan, M. A., et al. 2005, ApJ, 634, 142

Nagao, T., Murayama, T., Maiolino, R., et al. 2007, A\&A A, 468, 877

Schaerer, D. 2002, A\&A, 382, 28

Schaerer, D. 2003, A\&\&A, 397, 527 